\title{
Modeling the Dynamics of a Single-Species Model with Pollution Treatment in a Polluted Environment
}

\author{
Bing Liu, ${ }^{1}$ Shi Luan, ${ }^{2}$ and Yinghui Gao ${ }^{3}$ \\ ${ }^{1}$ College of Mathematics and Information Science, Anshan Normal University, Anshan, Liaoning 114007, China \\ ${ }^{2}$ Department of Mathematics, Liaoning Normal University, Dalian, Liaoning 116029, China \\ ${ }^{3}$ Department of Mathematics, Beihang University, Beijing 100083, China
}

Correspondence should be addressed to Bing Liu; liubing529@126.com

Received 19 November 2012; Accepted 25 December 2012

Academic Editor: Zhen Jin

Copyright (C) 2013 Bing Liu et al. This is an open access article distributed under the Creative Commons Attribution License, which permits unrestricted use, distribution, and reproduction in any medium, provided the original work is properly cited.

Without any question, environmental pollution is the main cause for the species extinction in recent times. In this paper, based on impulsive differential equation, the dynamics of a single-species model with impulsive pollution treatment at fixed time in a polluted environment is considered, in which we assume that the species is directly affected by the pollutants. Sufficient conditions for permanence and extinction of the species are given. The results show that the species is permanent when the impulsive period is less than some critical value, otherwise the species will be extinct. Although shortening the impulsive period can protect the species from extinction, it is expensive. To see how pollution treatment applications could be economical, we also establish a hybrid impulsive model involving periodic pollution treatment at fixed time with state-dependent pollution treatment applied when the pollution concentration reaches the given Environment Threshold (ET). It indicates that the hybrid method is the most effective method to protect the species from extinction. Numerical simulations confirm our theoretical results.

\section{Introduction}

With the globalization of economy and trade, environmental pollution increasingly becomes one of the most serious problems faced by all the countries in the world. For instance, the greenhouse effect, acid rain, and ozone depletion are the environmental effects of air pollution. Another problem that the whole world is concerned about is how to protect endangered species. Of the ecosystem types, the largest species extinction happened to tropical rainforests, including many species not investigated and named by people. Tropical forests are home to more than one-half of the Earth's known species. The scientists have predicted that in the following 30 years the tropical rainforests abundant in species will likely be destroyed by the contemporaries and that $5-10$ species in the tropical rainforests will likely tend to extinction. Environmental pollution is a major contributor to species extinction or near extinction and the further reduction of biodiversity. It affects the distribution of species in nature, reduces habitat animals live in, and causes mass migration of animal species and also has a negative effect on growth and reproduction of organisms, and when pollution is serious, organisms will change markedly in morphological specificity, their number, and the like. To preserve the ecological environment, we must curb environmental pollution.

After the pioneering theoretical work [1,2], some papers [3-9] have been researched on the effect of the continuous input pollution on the survival of a biological population. Considering the pollutants are emitted in regular pulses, Liu et al. have been focusing on the effect of impulsive pollution input on a single species [10-14] and two-species Lotka-Volterra competition system [15] and have obtained the conditions of the persistence and extinction for the population. Reference [16] investigated a logistic offshore fishery system with impulsive pollutant input in inshore areas and impulsive diffusion at different fixed time. They obtained the sufficient conditions of the existence of the positive periodic solution and the global asymptotic stability of both the trivial periodic solution and the positive periodic solution and gave the corresponding optimal harvesting strategy.

Based on the biological background mentioned above and with environmental pollution worsening, to protect 
endangered species, in this paper a single-species model with impulsive pollution treatment at fixed time in a polluted environment is established and studied. So far, no research has been done on how to use the mathematical model to study the effect of pollution control on population size. Besides, according to practical problems, we will consider constructing a hybrid impulsive single-species model combining periodic pollution treatment at fixed time with statedependent pollution treatment applied when the pollution concentration reaches the given Environment Threshold and gives specific biological explanations based on numerical simulations, and our conclusion will provide the theoretical decision-making basis for practical problems.

The organization of this paper is as follows. In the next section, a continuous model is proposed and its corresponding dynamic properties is obtained, then we establish a singlespecies model with impulsive pollution treatment at fixed time in a polluted environment. In Section 3, we obtain the conditions for permanence and extinction of such a system. The conditions of existence and stability of positive periodic solution of model are given in Section 4. In Section 5, we propose a hybrid impulsive single-species model with Environment Threshold and give biological explanations by numerical simulations. In the last section, we discuss our results and suggest future work.

\section{Model Formulation}

Firstly, we assume that the emission of pollutants to the environment is continuous and directly affects the survival of the species in such an environment. Let us consider the following single species model with the pollution effect:

$$
\begin{gathered}
\frac{d x(t)}{d t}=r x(t)\left(1-\frac{x(t)}{K}\right)-r_{1} x(t) c(t), \\
\frac{d c(t)}{d t}=\mu(t)-h c(t),
\end{gathered}
$$

where $x(t)$ is the density of the species at time $t$; $c(t)$ is the concentration of pollution in the environment at time $t ; K$ is the carrying capacity of environment; $r$ is the intrinsic growth rate of the species $x(t)$ in the absence of pollutants in the environment; $r_{1}$ is the dose-response parameter of species to the pollution concentration in the environment; $\mu(t)$ represents the exogenous rate of the pollutants input into the environment and is bounded, for the convenience, here we assume $\lim _{t \rightarrow \infty} \mu(t)=\mu ;-h c(t)$ represents the totality of losses from the environment including biological transformation, chemical hydrolysis, volatilization, microbial degradation, and photosynthetic degradation.

In this paper, we assume that the capacity of the environment is so large that the change of the concentration of pollution in the environment that comes from uptake and egestion by the species can be ignored.

Clearly in system (1), $x(t) \leq K$ and $\lim _{t \rightarrow \infty} c(t)=$ $\mu / h$. System (1) always has a species extinction equilibrium $E_{1}(0, \mu / h)$; if $r>r_{1} \mu / h$, there exists a unique positive equilibrium $E_{2}\left(K\left(r h-r_{1} \mu\right) / r h, \mu / h\right)$.
Let $E^{*}\left(x^{*}, c^{*}\right)$ be any equilibrium of system (1), then the characteristic equation of its equilibrium is

$$
Q=\left|\begin{array}{cc}
r-\frac{2 r x^{*}}{K}-r_{1} c^{*}-\lambda & -r_{1} x^{*} \\
0 & -h-\lambda
\end{array}\right|=0 .
$$

For the equilibrium $E_{1}(0, \mu / h),(2)$ is reduced to

$$
(\lambda+h)\left(\lambda+\frac{r_{1} \mu}{h}-r\right)=0 .
$$

If $r<r_{1} \mu / h$, both roots of (3) have negative real part, so $E_{1}$ is locally asymptotically stable.

If $r>r_{1} \mu / h$, one of the roots of (3) is positive, then $E_{1}$ is unstable saddle, and positive equilibrium point $E_{2}$ exists.

For this equilibrium $E_{2}\left(K\left(r h-r_{1} \mu\right) / r h, \mu / h\right)$, (2) is reduced to

$$
(\lambda+h)\left(\lambda+r-\frac{r_{1} \mu}{h}\right)=0 .
$$

If $r>r_{1} \mu / h$, both roots of (4) have negative real part, so $E_{2}$ is locally asymptotically stable.

Theorem 1. If $r<r_{1} \mu / h$, the species extinction equilibrium $E_{1}$ is globally asymptotic stable; whereas if $r>r_{1} \mu / h$, the positive equilibrium $E_{2}$ is globally asymptotic stable.

Proof. If $r<r_{1} \mu / h, E_{1}$ is locally stable. In this case, there is no any interior equilibrium in system (1), so there cannot be any periodic solutions in $R_{+}^{2}$ since a periodic solution must contain at least one equilibrium. By the Poincaré-Bendixson theory, $E_{1}$ is globally asymptotic stable.

If $r>r_{1} \mu / h$, then $E_{2}$ exists, and $E_{2}$ is locally asymptotically stable. Let $P(x, c), Q(x, c)$ be the right sides of (5), respectively, then we have

$$
\begin{gathered}
P(x, c)=r x(t)\left(1-\frac{x(t)}{K}\right)-r_{1} x(t) c(t), \\
Q(x, c)=\mu-h c(t) .
\end{gathered}
$$

Set Dulac function $B(x, c)=x^{-1} c^{-1}$, then we have

$$
\frac{\partial B P}{\partial x}+\frac{\partial B Q}{\partial c}=-\frac{r}{K c}-\frac{\mu}{x c^{2}}<0,
$$

which implies that there is no limit cycle in $R_{+}^{2}$, so the equilibrium $E_{2}$ is global asymptotically stable. This completes the proof.

Remark 2. Since $r<r_{1} \mu / h$ can be rewritten as $r<r_{1} c^{*}$, under this condition the growth rate of the species becomes $r-r_{1} c^{*}<0$. So the species $x(t)$ will tend to be extinct; that is, the concentration of pollution in the environment is violence against the survival of the species. If $r>r_{1} \mu / h$, it means that the growth rate of the species $r-r_{1} c^{*}>0$, and the species will be permanent and tend to the unique globally asymptotically stable positive equilibrium.

From the above theorem, we know if $r>r_{1} \mu / h, E_{1}(0, \mu / h)$ is globally asymptotically stable, that is, the species will 
tend to be extinct. In order to protect the population from extinction, we must take steps to control environmental pollution. Now based on the impulsive differential equation [10-22], we formulate the following single species model with impulsive pollution treatment at fixed time:

$$
\begin{aligned}
& \frac{d x(t)}{d t}=r x(t)\left(1-\frac{x(t)}{K}\right)-r_{1} x(t) c(t), \\
& \frac{d c(t)}{d t}=\mu-h c(t), \\
& \Delta x(t)=0, \quad \Delta c(t)=-\beta c(t), \quad t=n T, n \in Z^{+}, \\
& x(0)>0, \quad 0 \leq c(0) \leq 1,
\end{aligned}
$$

where $\Delta x(t)=x\left(t^{+}\right)-x(t), \Delta c(t)=c\left(t^{+}\right)-c(t) ; r, r_{1}, \mu, h$ are positive constants, and $Z^{+}=\{1,2, \ldots\} ; T$ is the period of impulsive effect and $\beta$ is the decrease rate of pollution concentration with the effects of pollution treatment. The other meanings of parameters are the same as those of model (1).

Remark 3. In this paper, we consider how to protect the species from extinction when the pollution concentration is large in the environment, so we assume that $r<r_{1} \mu / h$.

Remark 4. In the model (7), $c(t)$ is the concentration of pollution in the environment, so $0 \leq c(t) \leq 1$. To assure that $c_{0}(t)$ remains less than one, it is necessary that

$$
\mu \leq h \text {. }
$$

In the following, we always assume that (8) holds true.

\section{Extinction and Permanence}

In this section, we investigate the extinction and permanence of system (7).

First, we consider the following subsystem of system (7):

$$
\begin{gathered}
\frac{d c(t)}{d t}=\mu-h c(t), \quad t \neq n T, n \in Z^{+}, \\
\Delta c(t)=-\beta c(t), \quad t=n T, n \in Z^{+}, \\
0 \leq c(0) \leq 1,
\end{gathered}
$$

where $\Delta c(t)=c\left(t^{+}\right)-c(t)$

The following lemma is a corollary of Theorem 2.3 in [23].

Lemma 5 (see [23]). System (9) has a unique globally asymptotically stable positive $T$-periodic solution $\widetilde{c}(t)$, where

$$
\begin{gathered}
\tilde{c}(t)=\frac{\mu}{h}+\left[C^{*}-\frac{\mu}{h}\right] e^{-h(t-n T)}, \\
n T<t \leq(n+1) T, n \in Z^{+}, \\
C^{*}=\frac{\mu}{h}(1-\beta)\left(1-e^{-h T}\right)\left(1-(1-\beta) e^{-h T}\right)^{-1} .
\end{gathered}
$$

Therefore, system (7) has a species extinction periodic solution $(0, \widetilde{c}(t))$.
Remark 6. In model (9), $c(t)$ tends to a unique positive $T$ periodic solution $\widetilde{c}(t)$, which shows that periodic impulsive pollution treatment causes a periodic behavior of $c(t)$ in system (9).

Lemma 7. Let $f(T)=r T-r_{1} \mu T / h+r_{1} \mu \beta\left(1-e^{-h T}\right) /(1-(1-$ $\left.\beta) e^{-h T}\right) h^{2}$, then $f(T)=0$ has a unique positive root $T_{0}$, and one has

(1) if $0<T<T_{0}, f(T)>0$;

(2) if $T>T_{0}, f(T)<0$.

Proof. Obviously $f(0)=0$.

Calculate the derivative of $f(T)$, we have

$$
f^{\prime}(T)=r-\frac{r_{1} \mu}{h}+\frac{r_{1} \mu \beta\left(1-e^{-h T}\right)}{\left(1-(1-\beta) e^{-h T}\right)^{2} h} .
$$

Obviously, $f^{\prime}(0)=r>0$; Let $T \rightarrow+\infty, f^{\prime}(T) \rightarrow r-$ $r_{1} \mu / h<0$, since $f^{\prime \prime}(T)<0$. So we have $f^{\prime}(T)=0$ which has a unique positive root $T_{1}$. If $0<T<T_{1}$, we have $f^{\prime}(T)>0$, and then $f(T)>f(0)=0$ holds true for $0<T \leq T_{1}$; if $T>T_{1}$, we have $f^{\prime}(T)<0$. Since $T \rightarrow \infty, f(T) \rightarrow-\infty$, so we have $f(T)=0$, which has a unique positive root $T_{0}$, and $T_{0}>T_{1}$. Thus if $T_{1}<T<T_{0}$, we have $f(T)>0$; if $T>T_{0}$, we have $f(T)<0$. The proof is completed.

Now, we give sufficient conditions which drive $x(t)$ to be extinct.

Theorem 8. If $T>T_{0}$, then the species extinction periodic solution $(0, \tilde{c}(t))$ is globally asymptotically stable.

Proof. From Lemma 7, if $T>T_{0}, f(T)<0$. We choose sufficiently small $\varepsilon_{1}>0$ such that

$$
\delta=-r T+\frac{r_{1} \mu T}{h}-\frac{r_{1} \mu \beta\left(1-e^{-h T}\right)}{\left(1-(1-\beta) e^{-h T}\right) h^{2}}-r_{1} \varepsilon_{1} T>0 .
$$

From Lemma 5, we have $c(t) \rightarrow \widetilde{c}(t)$ as $t \rightarrow \infty$, that is, for $t$ being large enough,

$$
c(t)>\widetilde{c}(t)-\varepsilon_{1}
$$

holds. For the sake of simplicity, we assume that (13) holds true for all $t>0$. From system (7), we have

$$
\begin{aligned}
\frac{d x(t)}{d t} & =r x(t)\left(1-\frac{x(t)}{K}\right)-r_{1} c(t) x(t) \\
& \leq x(t)\left[r-r_{1}\left(\tilde{c}(t)-\varepsilon_{1}\right)\right],
\end{aligned}
$$

thus

$$
\begin{aligned}
& x((n+1) T) \\
& \quad \leq x(n T) \exp \left(\int_{n T}^{(n+1) T}\left[r-r_{1}\left(\widetilde{c}(t)-\varepsilon_{1}\right)\right] d t\right)
\end{aligned}
$$




$$
\begin{aligned}
& =x(n T) \exp \left(r T-\frac{r_{1} \mu T}{h}\right. \\
& \left.\quad+\frac{r_{1} \mu \beta\left(1-e^{-h T}\right)}{\left(1-(1-\beta) e^{-h T}\right) h^{2}}+r_{1} \varepsilon_{1} T\right) \\
& =x(n T) \exp (-\delta),
\end{aligned}
$$

so we get $x(n T) \leq x\left(0^{+}\right) \exp (-n \delta)$ and $x(n T) \rightarrow 0$ as $n \rightarrow \infty$. Therefore $x(t) \rightarrow 0$ as $t \rightarrow \infty$ since $0<x(t)<$ $x(n t) \exp \left(r_{0} T\right)$ for $n T<t \leq(n+1) T$. This completes the proof.

Definition 9. It is said that the species $x(t)$ of system (7) is permanent if there exists a positive number $\delta$ and a finite time $T$ such that $x(t) \geq \delta$ for all $t \geq T$.

On the permanence of system (7), we have the following result.

Theorem 10. The species $x(t)$ of system (7) is permanent if $0<$ $T<T_{0}$.

Proof. From Lemma 7, if $0<T<T_{0}, f(T)>0$. We choose sufficiently small $\delta_{1}, \varepsilon_{2}>0$ such that

$$
\sigma=r T-\frac{r_{1} \mu T}{h}+\frac{r_{1} \mu \beta\left(1-e^{-h T}\right)}{\left(1-(1-\beta) e^{-h T}\right) h^{2}}-r_{1} \varepsilon_{2} T-\frac{r \delta_{1} T}{K}>0 .
$$

From Lemma 5, we know for the above $\varepsilon_{2}>0$, there exists a $T^{*}>0$ such that

$$
c(t)<\widetilde{c}(t)+\varepsilon_{2}
$$

holds true for $t>T^{*}$.

We claim that the inequality $x(t) \leq \delta_{1}$ cannot hold for all $t>T^{*}$. Otherwise, we have

$$
\frac{d x(t)}{d t} \geq x(t)\left(r-r_{1}\left(\widetilde{c}(t)+\varepsilon_{2}\right)-\frac{r \delta_{1}}{K}\right), \quad t>T^{*} .
$$

Let $N_{1} \in Z^{+}$, and $N_{1} T \geq T^{*}$. Integrating inequality (18) on $(n T,(n+1) T]\left(n \geq N_{1}\right)$, we have

$$
\begin{aligned}
& x((n+1) T) \\
& \geq x(n T) \\
& \quad \times \exp \left(\int_{n T}^{(n+1) T}\left(r-r_{1}\left(\widetilde{c}(t)+\varepsilon_{2}\right)-\frac{r \delta_{1}}{K}\right) d t\right) \\
& =x(n T) \exp \left(r T-\frac{r_{1} \mu T}{h}\right. \\
& \quad+\frac{r_{1} \mu \beta\left(1-e^{-h T}\right)}{\left(1-(1-\beta) e^{-h T}\right) h^{2}} \\
& \left.\quad-r_{1} \varepsilon_{2} T-\frac{r \delta_{1} T}{K}\right) \\
& =x(n T) \exp (\sigma),
\end{aligned}
$$

so $x\left(\left(N_{1}+k\right) T\right) \geq x\left(\left(N_{1} T\right)^{+}\right) \exp (k \sigma) \rightarrow \infty(k \rightarrow \infty)$, which is a contradiction to the boundness of $x(t)$. Hence, there exists a $T_{2}>T^{*}$ such that $x\left(T_{2}\right)>\delta_{1}$. In the following, we will prove that $x(t) \geq \delta_{1} \exp (-\theta T)$ for all $t \geq T_{2}$, where

$$
\begin{array}{r}
\theta=\sup \left\{\left|r-r_{1}\left(\widetilde{c}(t)+\varepsilon_{2}\right)-\frac{r \delta_{1}}{K}\right|: t \in[0,+\infty)\right\}, \\
0<\theta<\infty .
\end{array}
$$

Otherwise, there exists a $t_{0}>T_{2}$ such that $x\left(t_{0}\right)<$ $\delta_{1} \exp \left(-\theta T_{2}\right)$. Then there exists a $t_{1} \in\left(T_{2}, t_{0}\right]$ such that $x\left(t_{1}\right)=\delta_{1}$ and $x(t)<\delta_{1}$ for $t \in\left(t_{1}, t_{0}\right]$. Let $N_{2} \in Z^{+}$, $t_{0} \in\left(t_{1}+N_{2} T, t_{1}+\left(N_{2}+1\right) T\right]$, we have

$$
\begin{aligned}
& \delta_{1} \exp (-\theta T) \\
& >x\left(t_{0}\right)=x\left(t_{1}\right) \exp \left(\int_{t_{1}}^{t_{0}}\left(r-r_{1} \widetilde{c}(t)-\frac{r x(t)}{K}\right) d t\right) \\
& \geq \delta_{1} \exp \left(\left(\int_{t_{1}}^{t_{1}+N_{2} T}+\int_{t_{1}+N_{2} T}^{t_{0}}\right)\right. \\
& \left.\quad \times\left(r-r_{1}\left(\widetilde{c}(t)+\varepsilon_{2}\right)-\frac{r \delta_{1}}{K}\right) d t\right) \\
& \geq \delta_{1} \exp \left(\int_{t_{1}+N_{2} T}^{t_{0}}\left(r-r_{1}\left(\widetilde{c}(t)+\varepsilon_{2}\right)-\frac{r \delta_{1}}{K}\right) d t\right) \\
& \geq \delta_{1} \exp (-\theta T),
\end{aligned}
$$

this is a contradiction. Let $\delta=\delta_{1} \exp (-\theta T)$, we can obtain $x(t) \geq \delta$ for all $t>T_{2}$. This completes the proof.

Remark 11. From Theorems 8 and 10, we obtain that the impulsive period $T_{0}$ is the threshold value which determines permanence or extinction of the species. It implies that if the impulsive period $T$ is less than the threshold value $T_{0}$, then the pollution in the environment is treated frequently and the concentration of the pollution in the environment decreases rapidly, and eventually the species will be permanent. In the next section, we will see in this case the species will tend to a positive globally asymptotically stable $T$-periodic solution; if the impulsive period $T$ is larger than the threshold value $T_{0}$, then the pollution in the environment is not treated in time and will drive the species to be extinct. Therefore, in order to protect the endangered species, we should ensure that $T<T_{0}$ holds true, which can be carried by several strategies, such as increasing the treatment rate $\beta$, increasing the frequency of pollution treatment, or controlling the emission of pollutants.

\section{Existence and Stability of Positive Periodic Solution of Model (7)}

Now we give the condition of existence and stability of positive periodic solution of model (7). First of all, we consider the following periodic logistic equation

$$
\frac{d u(t)}{d t}=u(t)(\alpha(t)-\beta(t) u(t))
$$


where $\alpha(t), \beta(t)$ are $T$-periodic continuous on $R$. A solution $u(t)$ of (22) is said to be positive, if $u(t)>0$ for all $t \geq 0$. It is easy to prove that the solution $u(t)$ of (22) is positive if and only if the initial $u(0)>0$.

Lemma 12 (see [24]). If $\beta(t)>0$ and $\int_{0}^{T} \alpha(t) d t>0$, then (22) has a unique positive T-periodic solution $\tilde{u}(t)$ which is globally asymptotically stable, that is; $u(t) \rightarrow \widetilde{u}(t)$ as $t \rightarrow \infty$ for any positive solution $u(t)$ of $(22)$.

We give a continuous function $a:[0,+\infty) \rightarrow R$, where $\alpha$ is a $T$-periodic continuous function; if $\lim _{t \rightarrow \infty}|a(t)-\alpha(t)|=$ 0 , we call $a$ is asymptotical $\alpha$. Consider the following unique solution of the Cauchy function:

$$
\begin{gathered}
\frac{d v(t)}{d t}=v(t)(a(t)-\beta(t) u(t)), \\
v(0)=v_{0},
\end{gathered}
$$

where $a(t)$ is asymptotical $\alpha, \beta(t)$ is a $T$-periodic continuous function.

Lemma 13 (see [24]). If $\beta(t)>0$ and $\int_{0}^{T} \alpha(t) d t>0$, for all $t \in R, \widetilde{u}(t)$ is a unique positive T-periodic solution of $(22), v(t)$ is the solution with initial value $v_{0}$ of (23), then for any initial values $v_{0}>0$, one has $v(t) \rightarrow \tilde{u}(t)$ as $t \rightarrow \infty$.

In the following, we give sufficient conditions of existence and stability of positive periodic solution by using Lemmas 12 and 13.

Theorem 14. For system (7), if $0<T<T_{0}$, then there exists a unique positive T-periodic solution $(\widetilde{x}(t), \widetilde{c}(t))$ which is globally asymptotically stable, that is, for any solution $(x(t), c(t))$ of system (7), we have

$$
(x(t), c(t)) \longrightarrow(\tilde{x}(t), \tilde{c}(t)) \text { as } t \longrightarrow \infty .
$$

Proof. From Lemma 5, we know that $c(t) \rightarrow \widetilde{c}(t)$ as $t \rightarrow \infty$, so we only prove if the condition of Theorem 14 holds, then in system (7) there exists a positive $T$-periodic, and $x(t) \rightarrow$ $\tilde{x}(t)$, as $t \rightarrow \infty$. In the following, we use the equivalent system of (7) to prove this theorem.

Consider the following equation:

$$
\frac{d \bar{x}(t)}{d t}=\bar{x}(t)\left(r-r_{1} \widetilde{c}(t)-\frac{r \bar{x}(t)}{K}\right) .
$$

For

$$
\int_{0}^{T}\left(r-r_{1} \tilde{c}(t)\right) d t=r T-\frac{r_{1} \mu T}{h}+\frac{r_{1} \mu \beta\left(1-e^{-h T}\right)}{\left(1-(1-\beta) e^{-h T}\right) h^{2}}>0,
$$

from Lemma 12, we know that in system (25) there exists a unique globally asymptotically positive $T$-periodic solution, denoted by $\widetilde{x}(t)$. Since $\widetilde{c}(t)$ is a $T$-periodic solution of system (7), so $\tilde{x}(t)$ is a $T$-periodic solution of the system (7). And $c(t) \rightarrow \widetilde{c}(t)$, as $t \rightarrow \infty$, we obtain that $c(t)$ is asymptotical $\widetilde{c}(t)$, and from Lemma 13, for any solution $x(t)$ of system (7), we have $x(t) \rightarrow \tilde{x}(t)$ as $t \rightarrow \infty$. The proof is completed.
Remark 15. From Theorem 14, we can see that the condition of the existence and stability of positive periodic solution of model (7) is consistent with the permanent condition. Therefore, if the impulsive period $T$ is smaller than the critical value $T_{0}$, through the effective pollution treatment, the concentration of pollution will not be sufficiently high to kill the species, and the species will be permanent and tends to a unique positive $T$-periodic solution of system (7) (see Figure 1(a)). Otherwise, the species will tend to extinction (see Figure 1(b)).

Finally, we evaluate the average density of the species in one period when system (7) has a positive periodic solution.

Since $\tilde{x}(t)$ is a positive periodic solution of system (7), we have $x(0)=x(T)$ which gives

$$
x(0)=x(0) \exp \left(\int_{0}^{T}\left(r-r_{1} \widetilde{c}(t)-\frac{r}{K \tilde{x}(t)}\right) d t\right),
$$

so

$$
\begin{aligned}
\frac{\int_{0}^{T} \tilde{x}(t) d t}{T}= & \frac{K \int_{0}^{T}\left(r-r_{1} \tilde{c}(t)\right) d t}{r T} \\
=K & \left(\frac{r T-r_{1} \mu T / h+r_{1} \mu \beta\left(1-e^{-h T}\right)}{\left(1-(1-\beta) e^{-h T}\right) h^{2}}\right) \\
& \times(r T)^{-1},
\end{aligned}
$$

which implies that the average number of species strictly depends on impulsive period and pollution emission rate. The population will be extinct if $T>T_{0}$.

\section{A Hybrid Impulsive Model with Environment Threshold}

Because of economic and technical reasons, although the factory takes measures to treat pollution at regular time before the pollutants are emitted to the environment, sometimes the concentration of the pollution in the environment is still large and threatens the survival of the species. In practice, the factory is required to make pollution treatment immediately when the concentration of pollution reaches the given Environment Threshold (ET). According to the above biological background, we establish the following hybrid impulsive model involving periodic pollution treatment at fixed time with state-dependent pollution treatment applied when the pollution concentration reaches the given Environment Threshold:

$$
\begin{aligned}
& \frac{d x(t)}{d t}=r x(t)\left(1-\frac{x(t)}{K}\right)-r_{1} x(t) c(t), \\
& \frac{d c(t)}{d t}=\mu-h c(t) \\
& \Delta x(t)=0, \\
& \Delta c(t)=-\beta_{1} c(t), \quad c=c_{0}, \\
& \Delta x(t)=0, \\
& \Delta c(t)=-\beta_{2} c(t), \quad t=n T, c \neq c_{0},
\end{aligned}
$$



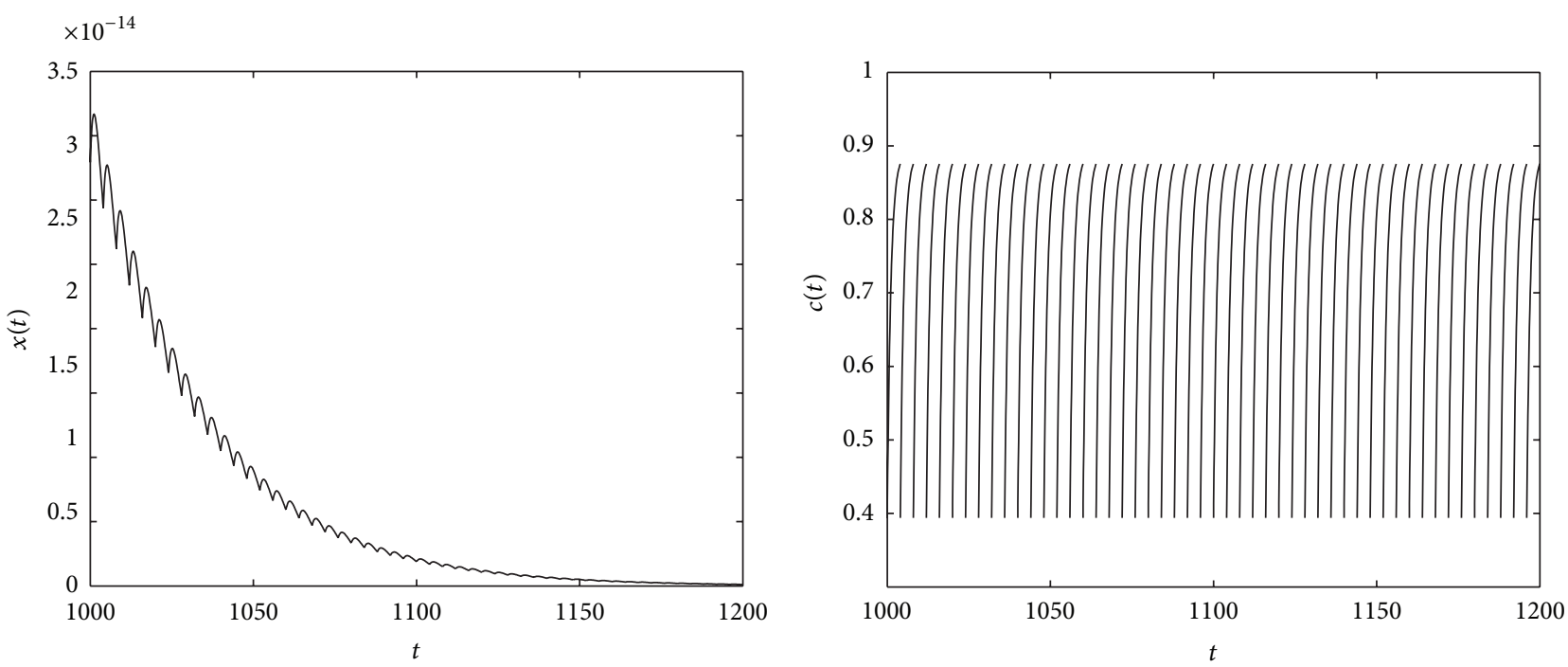

(a)
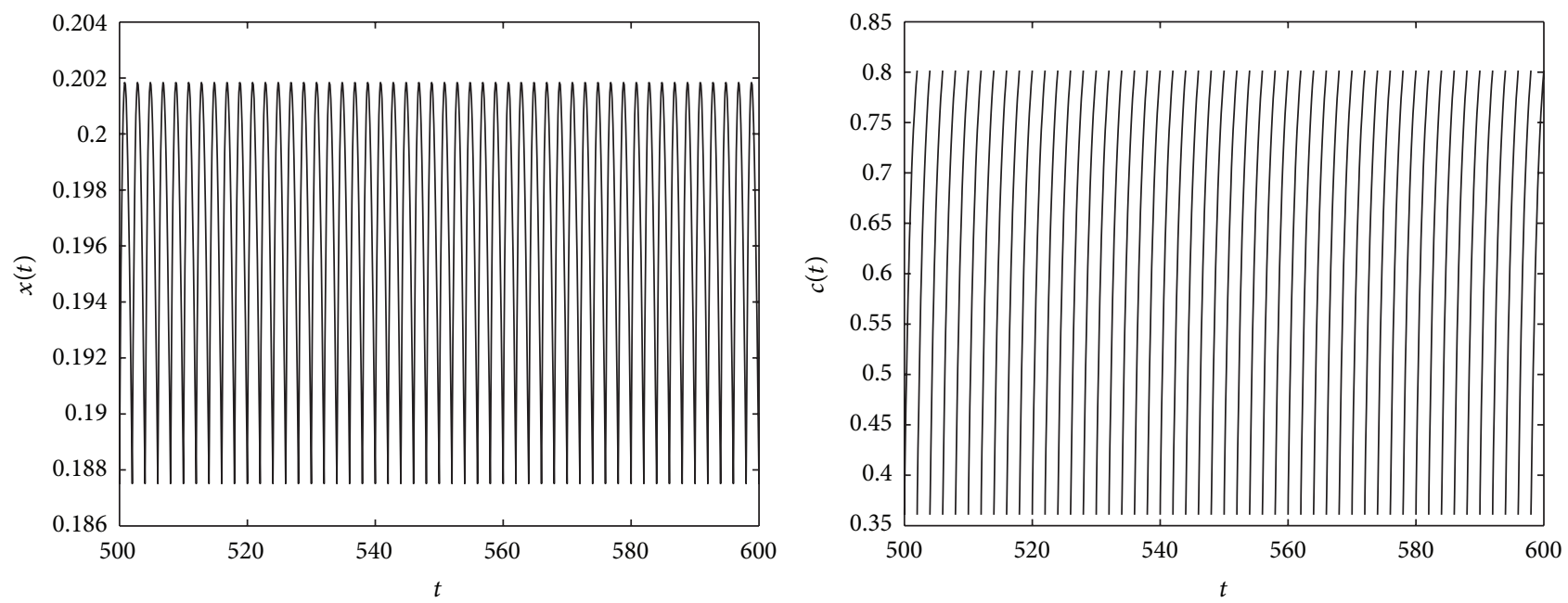

(b)

FIGURE 1: Time series of system (7). (a) Extinction solution of system (7) with parameters $r=0.5 ; K=2 ; r_{1}=0.7 ; \mu=0.8 ; h=0.9 ; T=4$; $\beta=0.55 ; x(0)=0.6 ; c(0)=0.5$. (b) $T$-periodic solution of system (7) with parameters $r=0.5 ; K=2 ; r_{1}=0.7 ; \mu=0.8 ; h=0.9 ; T=2$; $\beta=0.55 ; x(0)=0.6 ; c(0)=0.5$.

where $n T(n=1,2, \ldots)$ is periodic impulsive point series at which pollution treatment is taken, $c_{0}$ is the environmental threshold (ET) which threatens the survival of the species, and we assume that the initial concentration of pollution is less than $c_{0}$.

From the biological point of view, experimental methods in combination with the model approaches presented in this paper are the most effective methods. We have shown that the species $x(t)$ of system (7) with parameters in Figure 1(b) will tend to be extinct. If we only treat pollution at the fixed time to protect the species from extinction, we must shorten the impulsive period as shown in Figure 1(a). Sometimes it is difficult to put it into practice. Now we use the hybrid method to protect the species from extinction. Set ET $=0.8 ; \beta_{1}=0.4$; $\beta_{2}=0.55$; and other parameters are the same as those in Figure 1(b). When the concentration of pollution reaches ET, we treat pollution immediately. Then from Figure 2, we can see that the solution of system (29) will tend to a globally stable periodic solution, which means the species will be permanent.

\section{Discussion}

In this paper, we investigated a single-species model with impulsive pollution treatment at fixed time in a polluted environment. From Theorems 8 and 10, we can see that $T_{0}$ was the threshold condition under which the species became extinct or permanent. As long as $T$ was small enough and less than this threshold, the survival was predicted. Therefore, this threshold provided us with a way to choose the impulsive period $T$ and pollution treatment rate $\beta$ in order to protect the species from extinction. To see how pollution treatment 


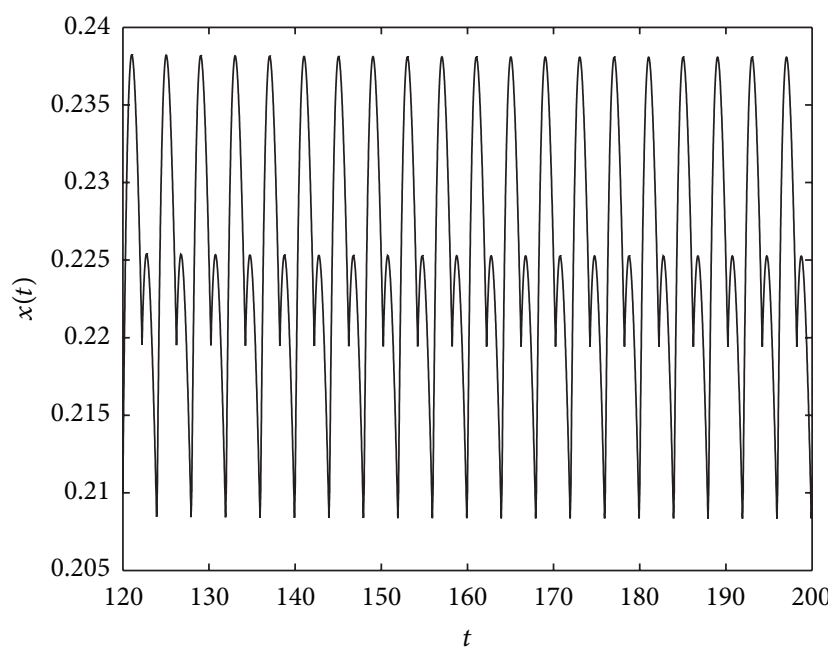

(a)

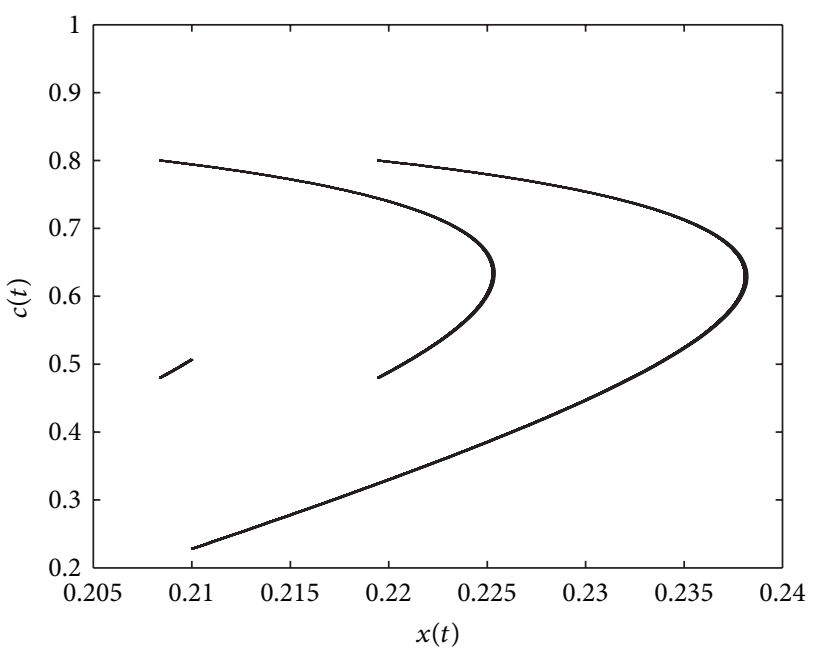

(b)

Figure 2: Dynamics of a hybrid impulsive model with parameters $r=0.5 ; K=2 ; r_{1}=0.7 ; \mu=0.8 ; h=0.9 ; T=4 ; \beta_{1}=0.4 ; \beta_{2}=0.55$; $\mathrm{ET}=0.8 ; x(0)=0.6 ; c(0)=0.5$. (a) Time series of the species $x(t)$. (b) Phase portrait.

applications could be economical, we also established a hybrid impulsive model involving periodic treatment at fixed time with state-dependent pollution treatment applied when the pollution concentration reached the given Environment Threshold. The results indicated that this hybrid method was the most effective method to protect the species from extinction.

Studying the effect of pollution treatment on the protection of the endangered species is at the very beginning. There is still much work to be done. For example, we have not studied the effect of pollution treatment on the single species with stage structure and time delay and two-species model in a polluted environment. We just leave these for the future work.

\section{Acknowledgments}

This work is supported by the National Natural Science Foundation of China (10971001, 111011021) and Science and Excellent Talents Support Project of Universities and Colleges in Liaoning China.

\section{References}

[1] T. G. Hallam, C. E. Clark, and R. R. Lassiter, "Effects of toxicants on populations: a qualitative approach. 1. Equilibrium environmental exposure," Ecological Modelling, vol. 18, no. 4, pp. 291-304, 1983.

[2] T. G. Hallam, C. E. Clark, and G. S. Jordan, "Effects of toxicants on populations: a qualitative approach. II. First order kinetics," Journal of Mathematical Biology, vol. 18, no. 1, pp. 25-37, 1983.

[3] Z. E. Ma, G. R. Cui, and W. D. Wang, "Persistence and extinction of a population in a polluted environment," Mathematical Biosciences, vol. 101, no. 1, pp. 75-97, 1990.

[4] P. D. N. Srinivasu, "Control of environmental pollution to conserve a population," Nonlinear Analysis. Real World Applications. An International Multidisciplinary Journal, vol. 3, no. 3, pp. 397-411, 2002.

[5] L. Huaping and M. Zhien, "The threshold of survival for system of two species in a polluted environment," Journal of Mathematical Biology, vol. 30, no. 1, pp. 49-61, 1991.

[6] D. Mukherjee, "Persistence and global stability of a population in a polluted environment with delay," Journal of Biological Systems, vol. 10, no. 3, pp. 225-232, 2002.

[7] F. Wang and Z. Ma, "Persistence and periodic orbits for an SIS model in a polluted environment," Computers \& Mathematics with Applications, vol. 47, no. 4-5, pp. 779-792, 2004.

[8] Y. Xiao and L. Chen, "How do the spatial structure and time delay affect the persistence of a polluted species," Applicable Analysis, vol. 82, no. 3, pp. 253-267, 2003.

[9] A. K. Pal and G. P. Samanta, "A single species population in a polluted environment," International Journal of Biomathematics, vol. 3, no. 2, pp. 187-204, 2010.

[10] B. Liu, L. Chen, and Y. Zhang, "The effects of impulsive toxicant input on a population in a polluted environment," Journal of Biological Systems, vol. 11, no. 3, pp. 265-274, 2003.

[11] F. Tao and B. Liu, "Dynamic behaviors of a single-species population model with birth pulses in a polluted environment," The Rocky Mountain Journal of Mathematics, vol. 38, no. 5, pp. 1663$1684,2008$.

[12] X.-Z. Meng, Q.-L. Zhao, and L.-S. Chen, "Global qualitative analysis of new monod type chemostat model with delayed growth response and pulsed input in polluted environment," Applied Mathematics and Mechanics, vol. 29, no. 1, pp. 75-87, 2008.

[13] B. Liu, L. Zhang, and Q. Zhang, "The effects of a single stagestructured population model with impulsive toxin input and time delays in a polluted environment," Applicable Analysis, vol. 88, no. 8, pp. 1143-1155, 2009.

[14] Z. Zhao, L. Chen, and X. Song, "Extinction and permanence of chemostat model with pulsed input in a polluted environment," Communications in Nonlinear Science and Numerical Simulation, vol. 14, no. 4, pp. 1737-1745, 2009.

[15] B. Liu and L. Zhang, "Dynamics of a two-species LotkaVolterra competition system in a polluted environment with pulse toxicant input," Applied Mathematics and Computation, vol. 214, no. 1, pp. 155-162, 2009. 
[16] B. Kang, B. Liu, and L. Xu, "Dynamics of an inshore-offshore fishery model with impulsive pollutant input in inshore area," Nonlinear Dynamics, vol. 67, no. 4, pp. 2353-2362, 2012.

[17] B. Liu, Y. Duan, and S. Luan, "Dynamics of an SI epidemic model with external effects in a polluted environment," Nonlinear Analysis. Real World Applications. An International Multidisciplinary Journal, vol. 13, no. 1, pp. 27-38, 2012.

[18] S. Sun and L. Chen, "Permanence and complexity of the ecoepidemiological model with impulsive perturbation," International Journal of Biomathematics, vol. 1, no. 2, pp. 121-132, 2008.

[19] Y. Xue, A. Kang, and Z. Jin, "The existence of positive periodic solutions of an eco-epidemic model with impulsive birth," International Journal of Biomathematics, vol. 1, no. 3, pp. 327-337, 2008.

[20] J. Jia and H. Cao, "Dynamic complexities of Holling type II functional response predator-prey system with digest delay and impulsive harvesting on the prey," International Journal of Biomathematics, vol. 2, no. 2, pp. 229-242, 2009.

[21] S. Tang, G. Tang, and R. A. Cheke, "Optimum timing for integrated pest management: modelling rates of pesticide application and natural enemy releases," Journal of Theoretical Biology, vol. 264, no. 2, pp. 623-638, 2010.

[22] W. Gao and S. Tang, "The effects of impulsive releasing methods of natural enemies on pest control and dynamical complexity," Nonlinear Analysis. Hybrid Systems. An International Multidisciplinary Journal, vol. 5, no. 3, pp. 540-553, 2011.

[23] Z. Hu and M. Han, "Periodic solutions and bifurcations of firstorder periodic impulsive differential equations," International Journal of Bifurcation and Chaos, vol. 19, no. 8, pp. 2515-2530, 2009.

[24] J. Lopez-Gomez, R. Ortega, and A. Tineo, "The periodic predator-prey Lotka-Volterra model," Advances in Differential Equations, vol. 1, no. 3, pp. 403-423, 1996. 


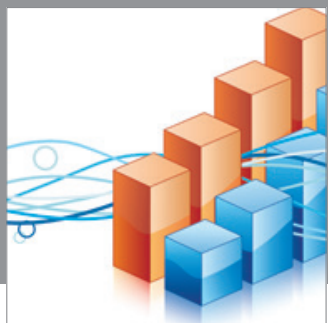

Advances in

Operations Research

mansans

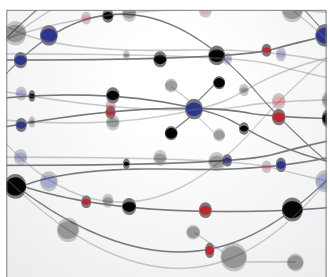

The Scientific World Journal
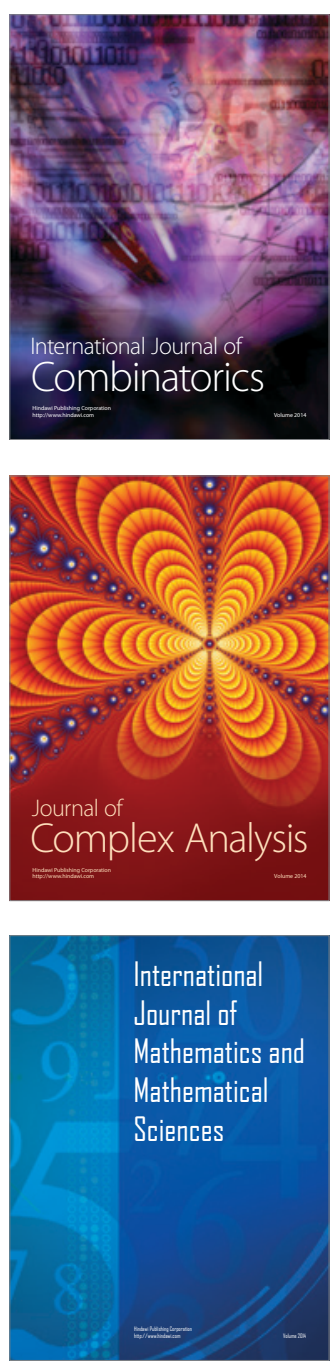
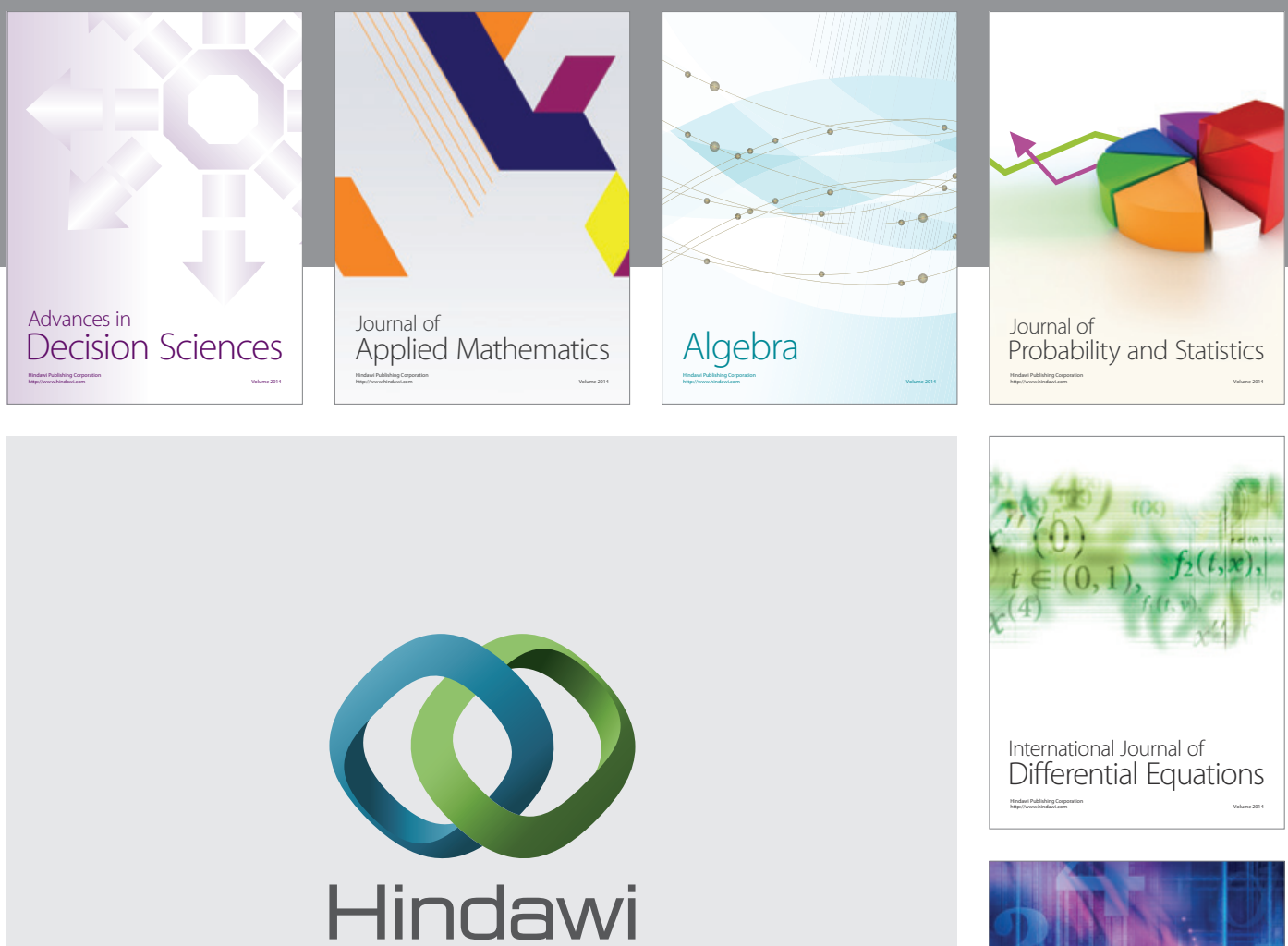

Submit your manuscripts at http://www.hindawi.com
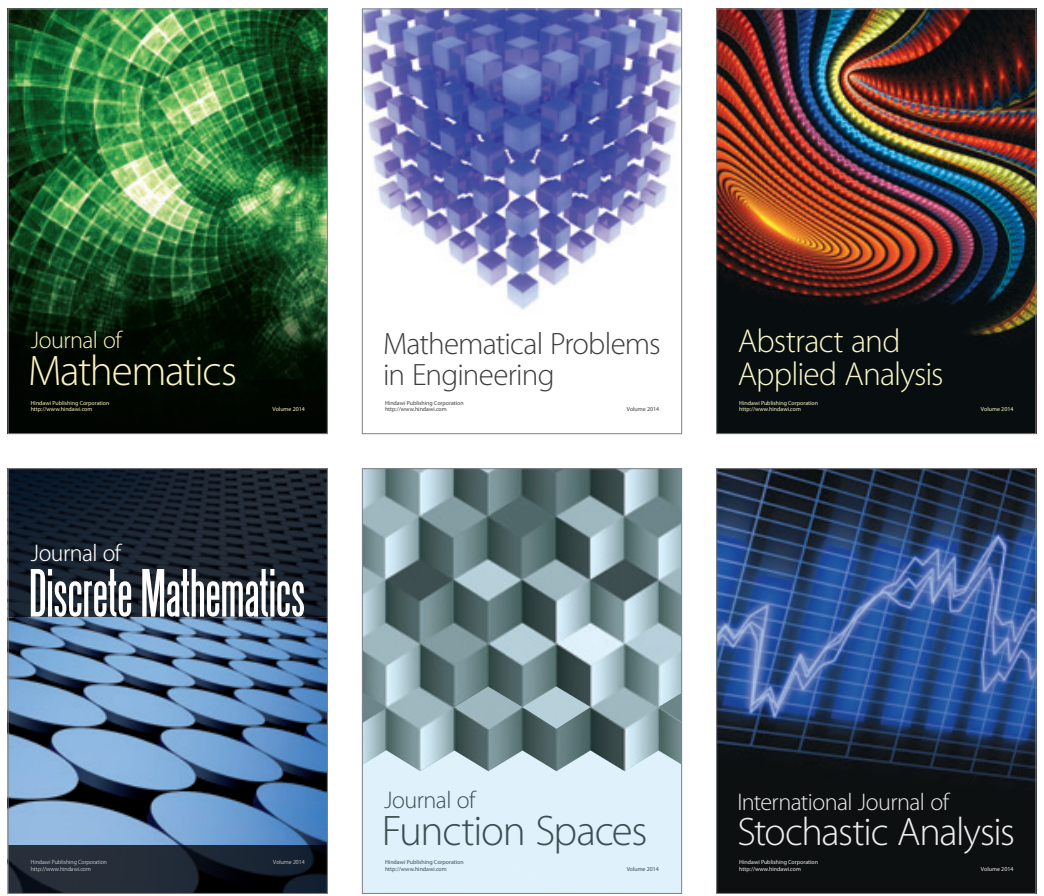

Journal of

Function Spaces

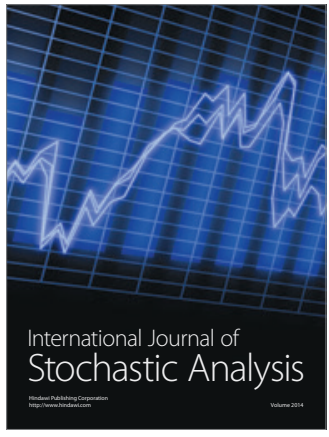

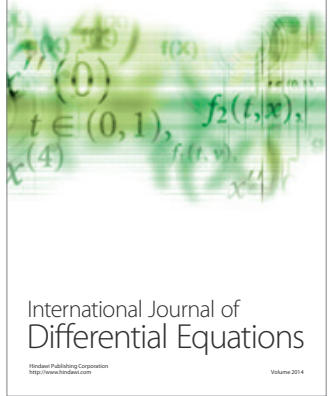
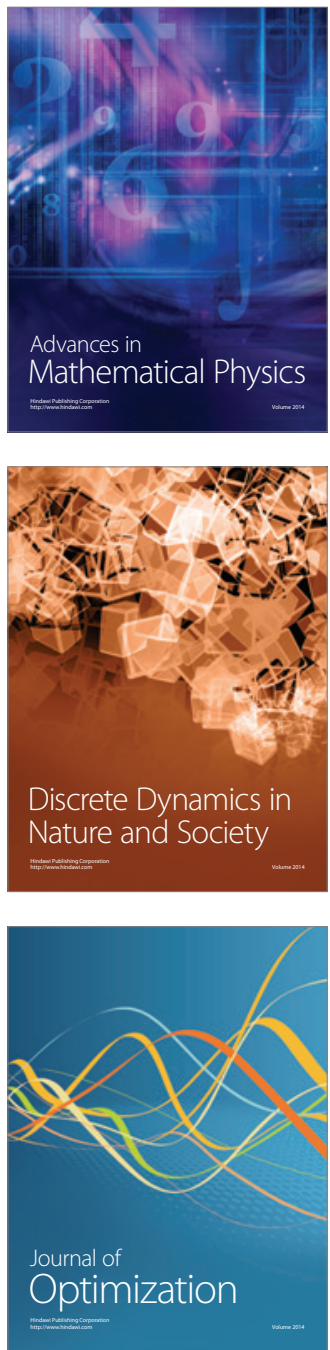